\title{
Quantitative Three-Dimensional Assessment of the Pharmacokinetic Parameters of Intra- and Peri-tumoural Tissues on Breast Dynamic Contrast-Enhanced Magnetic Resonance Imaging
}

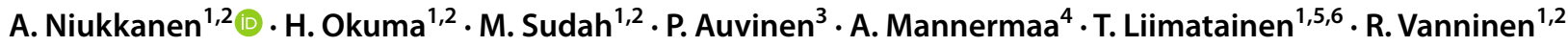

Received: 2 August 2020 / Revised: 2 July 2021 / Accepted: 17 August 2021 / Published online: 10 September 2021

(c) The Author(s) 2021

\begin{abstract}
We aimed to assess the feasibility of three-dimensional (3D) segmentation and to investigate whether semi-quantitative dynamic contrast-enhanced magnetic resonance imaging (DCE-MRI) parameters are associated with traditional prognostic factors for breast cancer. In addition, we evaluated whether both intra-tumoural and peri-tumoural DCE parameters can differentiate the breast cancers that are more aggressive from those that are less aggressive. Consecutive patients with newly diagnosed invasive breast cancer and structural breast MRI (3.0 T) were included after informed consent. Fifty-six patients (mean age, 57 years) with mass lesions of $>7 \mathrm{~mm}$ in diameter were included. A semi-automatic image post-processing algorithm was developed to measure 3D pharmacokinetic information from the DCE-MRI images. The kinetic parameters were extracted from time-signal curves, and the absolute tissue contrast agent concentrations were calculated with a reference tissue model. Markedly, higher intra-tumoural and peri-tumoural tissue concentrations of contrast agent were found in high-grade tumours $(n=44)$ compared to low-grade tumours $(n=12)$ at every time point $(P=0.006-0.040)$, providing positive predictive values of $90.6-92.6 \%$ in the classification of high-grade tumours. The intra-tumoural and peri-tumoural signal enhancement ratios correlated with tumour grade, size, and Ki67 activity. The intra-observer reproducibility was excellent. We developed a model to measure the 3D intensity data of breast cancers. Low- and high-grade tumours differed in their intra-tumoural and peri-tumoural enhancement characteristics. We anticipate that pharmacokinetic parameters will be increasingly used as imaging biomarkers to model and predict tumour behavior, prognoses, and responses to treatment.
\end{abstract}

Keywords Dynamic contrast-enhanced $\cdot$ MRI $\cdot$ Breast cancer $\cdot$ Peri-tumoural $\cdot$ Reference tissue model $\cdot$ Pharmacokinetic

\author{
A. Niukkanen \\ antoni@uef.fi \\ H. Okuma \\ hidemi.okuma@kuh.fi \\ M. Sudah \\ mazen.sudah@kuh.fi \\ P. Auvinen \\ paivi.auvinen@kuh.fi \\ A. Mannermaa \\ arto.mannermaa@uef.fi \\ T. Liimatainen \\ timo.liimatainen@oys.fi \\ R. Vanninen \\ ritva.vanninen@kuh.fi
}

1 Department of Clinical Radiology, Diagnostic Imaging Center, Kuopio University Hospital, PO BOX 100, 70029 KYS, Kuopio, Finland

2 Institute of Clinical Medicine, School of Medicine, Clinical Radiology, University of Eastern Finland, Kuopio, Finland

3 Institute of Clinical Medicine, School of Medicine, Oncology, University of Eastern Finland, Kuopio, Finland

4 Institute of Clinical Medicine, Pathology and Forensic Medicine, University of Eastern Finland, Kuopio, Finland

5 Physics and Technology, Research Unit of Medical Imaging, University of Oulu, Oulu, Finland

6 Department of Radiology, Oulu University Hospital, Oulu, Finland 


\section{Introduction}

Dynamic contrast-enhanced magnetic resonance imaging (DCE-MRI) has been used extensively in oncological imaging for decades. DCE-MRI allows for malignant and benign tumours in the breast to be distinguished based on differences in the contrast agent enhancement patterns, and thus, the method improves diagnostic accuracy, with proven importance in differential diagnostics and preoperative evaluation [1]. To evaluate the enhancement characteristics of lesions over time, a time intensity curve (TIC) is calculated by defining the region of interest (ROI) on the most suspicious region of enhancement within a lesion as instructed by the American College of Radiology Breast Imaging Reporting and Data System (ACR BI-RADS) [2]. The initial phase of the TIC is divided into slow, medium, and fast enhancement, and the delayed phase is divided into the persistent, plateau, and washout curves.

In DCE-MRI, the distribution of gadolinium contrast agent is compared between the vasculature and the intracellular and extracellular spaces at different time points. Therefore, the vascular density and the permeability of the vasculature in these tissues can be assessed, and the shape of the TIC can be determined. High permeability is linked to vascular leaking, which is attributed to tumour-growthrelated angiogenesis [3]. Therefore, DCE-MRI-based parameters may be associated with the histopathological properties of tumours and may indicate their relative aggressiveness, and DCE-MRI may allow for higher precision pathophysiological assessment of tumours and the monitoring of therapeutic interventions [4].

Early breast cancer DCE-MRI studies detected an association between the TIC type, rapid initial enhancement, and microvessel density [5]. The link between imaging and histopathological characteristics was strong enough to subclassify malignant breast tumours based on DCE-MRI [6]. Since these early discoveries, many new tools have been developed to extract additional information from medical images, ranging from hardware to deep learning-based image analysis solutions. This has led to the invention of radiomics, which uses data characterization algorithms to compute image features, called 'radiomic features' [7, 8]. These quantitative image features can accurately predict the subtype and genotypes of breast cancer [9, 10]. Furthermore, as cancer treatment becomes more individualized, DCE characteristics combined with other imaging parameters may provide more extensive prognostic information [11].

Most DCE-MRI studies have focused on measuring the intra-tumoural and background parenchymal features in individual two-dimensional (2D) slices with manually drawn ROIs [12-14]. More recently, it has been suggested that features extracted from peri-tumoural tissues could offer additional markers by reflecting angiogenic activity [15]. However, there is as yet no consensus on the optimal method of segmenting the peri-tumoural volume.

In this single-institution observational study, a semiautomatic method was developed to segment the intratumoural and peri-tumoural volumes of breast cancers threedimensionally in order to analyze their pharmacokinetic properties. Our main objectives were to assess the feasibility of three-dimensional (3D) segmentation in a consecutive clinical population and to investigate whether semiquantitative DCE parameters are associated with traditional prognostic factors. We also hypothesized that both intratumoural and peri-tumoural DCE parameters can be used to differentiate the breast cancers that are more aggressive from those that are less aggressive.

\section{Materials and Methods}

\section{Study Design and Patients}

This study was based on a database of 262 consecutive breast cancer patients prospectively included in a translational breast cancer study in 2011-2014 at our tertiary university hospital. Of these patients, the current study included those women who met the following criteria: [1] newly diagnosed invasive breast cancer; [2] pre-operative bilateral 3.0 T breast MRI; [3] mass lesions clearly demarcated on DCE-MRI; [4] healthy contralateral breast; [5] no previous history of cancer or breast operations; and [6] minimal tumour diameter of $>7 \mathrm{~mm}$. This minimal diameter was selected as the threshold for inclusion to avoid possible partial volume effects in smaller structures. At our institution, breast MRI is performed according to the guidelines of the European Society of Breast Cancer Specialists working group [16]. Fifty-six patients fulfilled the inclusion criteria and are the study cohort (Table 1). Written informed consent was obtained from all the patients before any procedure. The study was approved by the Research Ethics Board of our tertiary hospital and patients provided written informed consent. All clinical investigations were conducted according to the relevant guidelines and the principles expressed in the Declaration of Helsinki.

\section{Breast MRI}

MRI examinations were performed in the prone position with a seven-element phased-array coil dedicated to breast imaging (Philips Achieva 3.0-T TX, Philips N.V., Eindhoven, Netherlands). The clinical structural breast MRI protocol consisted of T2-weighted and 
Table 1 Patient profiles and tumour characteristics

\begin{tabular}{|c|c|}
\hline Characteristic & $N(\%)$ \\
\hline Patients/lesions & $56 / 56$ \\
\hline Age (years) & $56.6 \pm 11.0$ \\
\hline \multicolumn{2}{|l|}{ Menopause status } \\
\hline Premenopause & $19(33.9)$ \\
\hline Postmenopause & $37(66.1)$ \\
\hline \multicolumn{2}{|l|}{ Tumour stage } \\
\hline pT1 & $34(60.7)$ \\
\hline pT2 & $21(37.5)$ \\
\hline pT3 & $1(1.8)$ \\
\hline pT4 & $0(0)$ \\
\hline \multicolumn{2}{|l|}{ Axillary node classification } \\
\hline pNO & $33(58.9)$ \\
\hline pN1 & $16(28.6)$ \\
\hline $\mathrm{pN} 2$ & $5(8.9)$ \\
\hline $\mathrm{pN} 3$ & $2(3.6)$ \\
\hline \multicolumn{2}{|l|}{ Histological grade } \\
\hline G1 & $12(21.4)$ \\
\hline $\mathrm{G} 2$ & $27(48.4)$ \\
\hline G3 & $17(30.4)$ \\
\hline \multicolumn{2}{|c|}{ Human epidermal growth factor receptor 2} \\
\hline Positive & $45(80.4)$ \\
\hline Negative & $11(19.6)$ \\
\hline \multicolumn{2}{|l|}{ Oestrogen receptor } \\
\hline Positive & $48(85.7)$ \\
\hline Negative & $8(14.3)$ \\
\hline \multicolumn{2}{|l|}{ Progesterone receptor } \\
\hline Positive & $44(78.6)$ \\
\hline Negative & $12(21.4)$ \\
\hline \multicolumn{2}{|l|}{ Ki67 expression } \\
\hline$<20 \%$ & $26(46.4)$ \\
\hline$\geq 20 \%$ & $30(53.6)$ \\
\hline \multicolumn{2}{|l|}{ Tumour type } \\
\hline Ductal (no special type) & $45(80.4)$ \\
\hline Lobular & $8(14.3)$ \\
\hline Others & $3(5.4)$ \\
\hline
\end{tabular}

non-contrast- and contrast-enhanced 3D T1-weighted sequences and diffusion-weighted imaging. Dynamic contrast-enhanced fat-saturated 3D T1-weighted sequences $\left(\mathrm{TR}=4.70 \mathrm{~ms}\right.$; $\mathrm{TE}=2.30 \mathrm{~ms}$; flip angle $10^{\circ}$; in-plane pixel size $0.96 \times 0.96 \mathrm{~mm} ; 180$ slices; slice thickness $1 \mathrm{~mm}$; scanning time $58.5 \mathrm{~s}$ ) were used in this study, enhanced with an injection $(0.1 \mathrm{~mL} / \mathrm{kg}, 3 \mathrm{~mL} / \mathrm{s})$ of gadoterate meglumine $(376.9 \mathrm{mg} / \mathrm{mL})$, followed by a saline chaser. An initial pre-contrast and six (total) post-contrast sequences were used for segmentation and the subsequent assessment of the pharmacokinetic parameters.

\section{Assessment of Pharmacokinetic Parameters}

A single observer (AN) with 4 years of experience in breast MRI analysis performed all DCE analyses, in consultation with a breast radiologist (MS) with 25 years of experience in breast radiology. For this study, the open-source image-processing package Fiji (http://fiji.sc/Fiji; in the public domain, [version $1.52 \mathrm{p}]$ ) was used for the segmentation and analysis of the intratumoural and peri-tumoural pharmacokinetic properties [17]. A script was written to automate every step, except for the initial cropping of the tumour and the colour-changing procedure discussed below. Automating the process allowed a dataset of 1260 images to be analyzed in $1 \mathrm{~min}$, excluding the time required to import the images from the image database because this is heavily dependent on the system used. Every patient's 3D DCE-MRI image stack was analyzed separately. The computer used for the analysis had an Intel Core i3-6100 CPU and 16 GB of RAM. The code used in this study does not use graphics card acceleration.

\section{Image Analysis}

\section{k-Means Segmentation}

A $k$-means segmentation technique [18] was used to label all voxels separately at every time point after the whole DCEMRI image stack was deinterleaved. This method involves an unsupervised algorithm that assigns a membership to each voxel. Voxels are assigned to a cluster based on their proximity to the cluster centroids. Essentially different tissue types and backgrounds are defined based on their locations and intensity values. The clustering plug-in is based on a validated $k$-means algorithm [19].

\section{Parameter Acquisition}

Because the method presented has multiple steps, a script was written to increase its effectiveness and to avoid tedious manual effort. The main pipeline is presented in Fig. 1. First, the image stack was imported into Fiji and an ROI was selected manually. The ROI selection was based on placing a rectangle over the tumour area in the MR slice with the largest tumour area and then stretched laterally and horizontally to ensure complete selection of the tumour. The whole DCE image stack was then deinterleaved into stacks that represented individual time points; in this case, seven stacks were made. The last post-contrast image stack was used to create a 2D maximum intensity projection (MIP), which was then segmented with $k$-means clustering. Initial clustering was carried out with two clusters with a cluster 

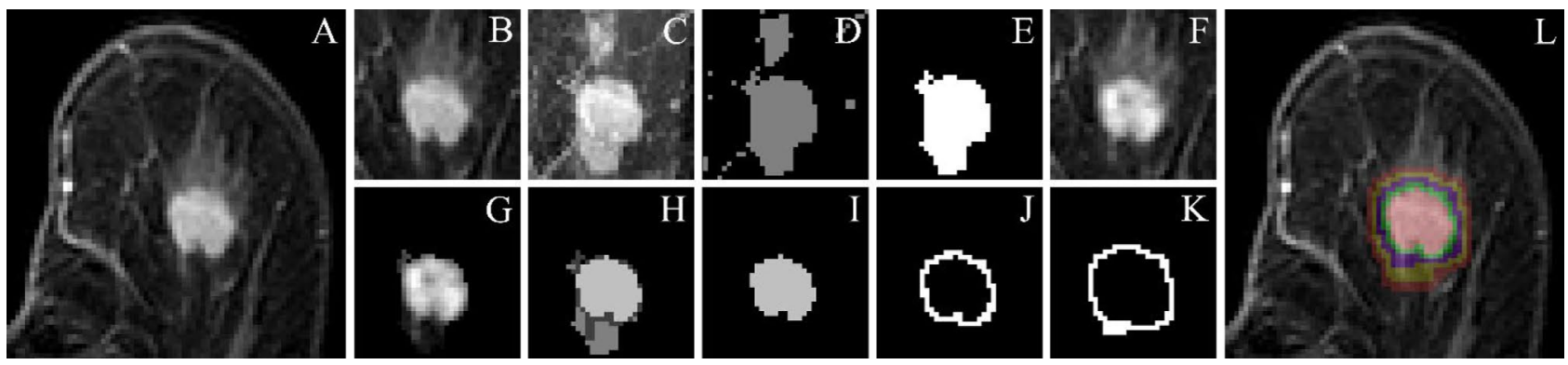

Fig. 1 Illustration of the method. A Original DCE-MRI stack. B Cropped tumour from the last time point of post-contrast imaging. $\mathbf{C}$ Maximum intensity projection (MIP) of cropped stack. D $k$-meansclustered MIP. E Small objects removed. F First time point of postcontrast image stack. G First post-contrast image stack after applica-

center tolerance of 0.0001 , a random seed, and the image stack was interpreted as 3D. The clustered MIP was used as a mask to approximate the tumour borders coarsely by removing all the non-enhanced structures from the original 3D image stack.

After the MIP mask was applied, the $k$-means clustering algorithm was used again with four clusters to further define the edges of the tumour. The image stacks were clustered as 3D volumes into four clusters. The clusters not including tumoural tissue were removed with a colour-changing operation. Because the results of $k$-means clustering were unpredictable, this step required observer intervention. After these steps, all extra-tumoural structures were removed with a plug-in, which removed all but the largest object in the 3D stack [20]. Finally, a precise tumour volume was left. To measure the peri-tumoural volume, four stepwise-dilated tumour volumes were created and made hollow so that each of the peri-tumoural shells was one voxel thick. The shells were identified from the tumour side outwards as shells 1 , 2,3 , and 4 .

The intensities of the healthy background fibroglandular tissues were measured by cropping the healthy breast tissue and deinterleaving the DCE-MRI image stack. The pre-contrast stack was $k$-means clustered and the colour values of the resulting clusters were modified so that only the fibroglandular tissue was left. Because the skin and fibroglandular tissues have similar intensities, they were assigned to the same cluster when $k$-means clustering was applied. However, the skin volume was removed from the selection by eroding the clustered volume. The erosion tool works by eroding the selected volume from the outside. Because the fibroglandular tissue is in the inner part of the breast, a fixed amount of erosion could be used to remove the skin without removing FGT in any significant amount. For adipose tissue measurements, a similar $k$-means clustering method tion of the edited MIP mask. $\mathbf{H} k$-means-clustered volume under the MIP-mask. I Tumour volume. J Peri-tumoural volume of "shell 1". K Three-dimensional dilated peri-tumoural volume of "shell 2". L Tumour and peri-tumoural shell masks applied to the original DCEMRI stack

was used to extract the intensity data. These volumes of the different tissues were then used as masks to measure the intensities in the unmodified DCE-MRI stacks using a 3D intensity measurement tool [21].

\section{Mathematical Models}

DCE-MRI data are typically interpreted based on the relationship between the intensity change and the contrast agent concentration. Although the concentration can be calculated using arterial input function methods, for this clinical study, a reference tissue method was used [22, 23].

\section{Reference Tissue Method}

The MRI signal intensity $(S(t))$ at time $t$ was compared with the initial T1-weighted image intensity $(S(0))$ to obtain the contrast agent concentration $(C(t))$ in the selected tissue. The contrast agent concentration was quantified with Eq. 1 [24]. An approximate value was calculated by taking measurements from a reference tissue and combining the data with baseline $T_{1}$ relaxation time values taken from the literature.

$C_{t}(t) \approx \frac{1}{r_{1}} \times \frac{1}{T_{1}(0)_{\text {reference tissue }} S(0)_{\text {reference tissue }}} \times(S(t)-S(0))$

In our study, the adipose tissue of breasts was used as the reference tissue and a value of $366 \mathrm{~ms}$ (taken from the literature) was used for the adipose tissue baseline $T_{1}$ relaxation time [25]. The relaxivity coefficient $\left(r_{1}\right)$ of the contrast agent was assumed to be constant $\left(3.43 \mathrm{mM}^{-1} \mathrm{~s}^{-1}\right.$ at $3 \mathrm{~T}$ ) [26]. Differences in the tissue concentrations of the contrast agent were measured with area under the curve (AUC) values and absolute concentrations $(\mathrm{mmol} / \mathrm{L})$. 


\section{Quantitative Kinetic Parameters}

In addition to the tissue concentrations over time, the quantitative kinetic parameters were also extracted from the TICs. The initial percentage enhancement $\left(E_{1}\right)$, peak percentage enhancement $\left(E_{\text {peak }}\right)$, and signal enhancement ratio (SER) were calculated as follows [27]:

$E_{1}=100 \% \times \frac{\left(S_{1}-S_{0}\right)}{S_{0}}$

$E_{\text {peak }}=100 \% \times \frac{\left(S_{\text {peak }}-S_{0}\right)}{S_{0}}$

$S E R=\frac{\left(S_{1}-S_{0}\right)}{\left(S_{\text {last }}-S_{0}\right)}$ where $S_{1}$ is the signal intensity in the volume of interest at the first contrast-enhanced point, $S_{\text {peak }}$ is the peak signal intensity, $S_{0}$ is the unenhanced signal intensity in the volume of interest, and $S_{\text {last }}$ is the signal intensity in the volume of interest at the last contrast enhancement point.

\section{Statistical Analysis}

All statistical analyses were performed with IBM SPSS Statistics for Windows, version 22 (IBM Corp., Armonk, NY, USA). Continuous variables are presented as mean \pm standard deviation (SD) and categorical variables as absolute values and percentages. Two-tailed Student's $t$-test was used to assess the differences between tumours of different grades. Pearson's correlation coefficient was used to test the associations between continuous variables. The reproducibility of the method was tested with Cronbach's $\alpha$ and intra-class
Table 2 Mean contrast agent concentrations $(\mathrm{mmol} / \mathrm{L})$ of grade $1(n=12)$ and grades $2-3$ $(n=44)$ tumours at different time points and in different regions of interest

\begin{tabular}{|c|c|c|c|c|c|c|}
\hline \multirow[t]{2}{*}{ Region } & \multirow[t]{2}{*}{ Time point } & \multicolumn{2}{|c|}{ Grade 1} & \multicolumn{2}{|c|}{ Grades 2 and 3} & \multirow[t]{2}{*}{$P$} \\
\hline & & Mean & SD & Mean & SD & \\
\hline \multirow[t]{6}{*}{ Intra-tumoural } & 2 & 2.47 & 1.61 & 4.15 & 1.86 & .006 \\
\hline & 3 & 3.05 & 1.75 & 4.84 & 1.99 & .006 \\
\hline & 4 & 3.40 & 2.01 & 4.97 & 1.96 & .017 \\
\hline & 5 & 3.51 & 1.98 & 4.95 & 1.89 & .024 \\
\hline & 6 & 3.46 & 1.73 & 4.92 & 1.83 & .016 \\
\hline & 7 & 3.54 & 1.76 & 4.86 & 1.81 & .029 \\
\hline \multirow[t]{6}{*}{ Peri-tumoural shell 1} & 2 & 0.83 & 0.47 & 1.37 & 0.68 & .013 \\
\hline & 3 & 1.28 & 0.63 & 1.93 & 0.85 & .016 \\
\hline & 4 & 1.54 & 0.68 & 2.23 & 0.94 & .021 \\
\hline & 5 & 1.73 & 0.74 & 2.43 & 1.00 & .029 \\
\hline & 6 & 1.84 & 0.78 & 2.56 & 1.06 & .032 \\
\hline & 7 & 1.97 & 0.80 & 2.68 & 1.09 & .040 \\
\hline \multirow[t]{6}{*}{ Peri-tumoural shell 2} & 2 & 0.39 & 0.21 & 0.60 & 0.34 & .056 \\
\hline & 3 & 0.65 & 0.31 & 0.92 & 0.43 & .053 \\
\hline & 4 & 0.79 & 0.30 & 1.08 & 0.51 & .068 \\
\hline & 5 & 0.92 & 0.33 & 1.22 & 0.56 & .078 \\
\hline & 6 & 1.02 & 0.37 & 1.31 & 0.60 & .108 \\
\hline & 7 & 1.09 & 0.36 & 1.41 & 0.63 & .106 \\
\hline \multirow[t]{6}{*}{ Peri-tumoural shell 3} & 2 & 0.34 & 0.19 & 0.51 & 0.32 & .074 \\
\hline & 3 & 0.54 & 0.26 & 0.78 & 0.40 & .054 \\
\hline & 4 & 0.66 & 0.27 & 0.91 & 0.46 & .083 \\
\hline & 5 & 0.77 & 0.28 & 1.02 & 0.50 & .102 \\
\hline & 6 & 0.83 & 0.29 & 1.08 & 0.52 & .105 \\
\hline & 7 & 0.89 & 0.31 & 1.15 & 0.54 & .116 \\
\hline \multirow[t]{6}{*}{ Peri-tumoural shell 4} & 2 & 0.31 & 0.19 & 0.46 & 0.29 & .089 \\
\hline & 3 & 0.49 & 0.26 & 0.70 & 0.37 & .059 \\
\hline & 4 & 0.60 & 0.27 & 0.85 & 0.42 & .082 \\
\hline & 5 & 0.70 & 0.29 & 0.92 & 0.45 & .112 \\
\hline & 6 & 0.73 & 0.28 & 0.87 & 0.47 & .092 \\
\hline & 7 & 0.80 & 0.31 & 1.03 & 0.49 & .115 \\
\hline
\end{tabular}

$S D$ standard deviation 


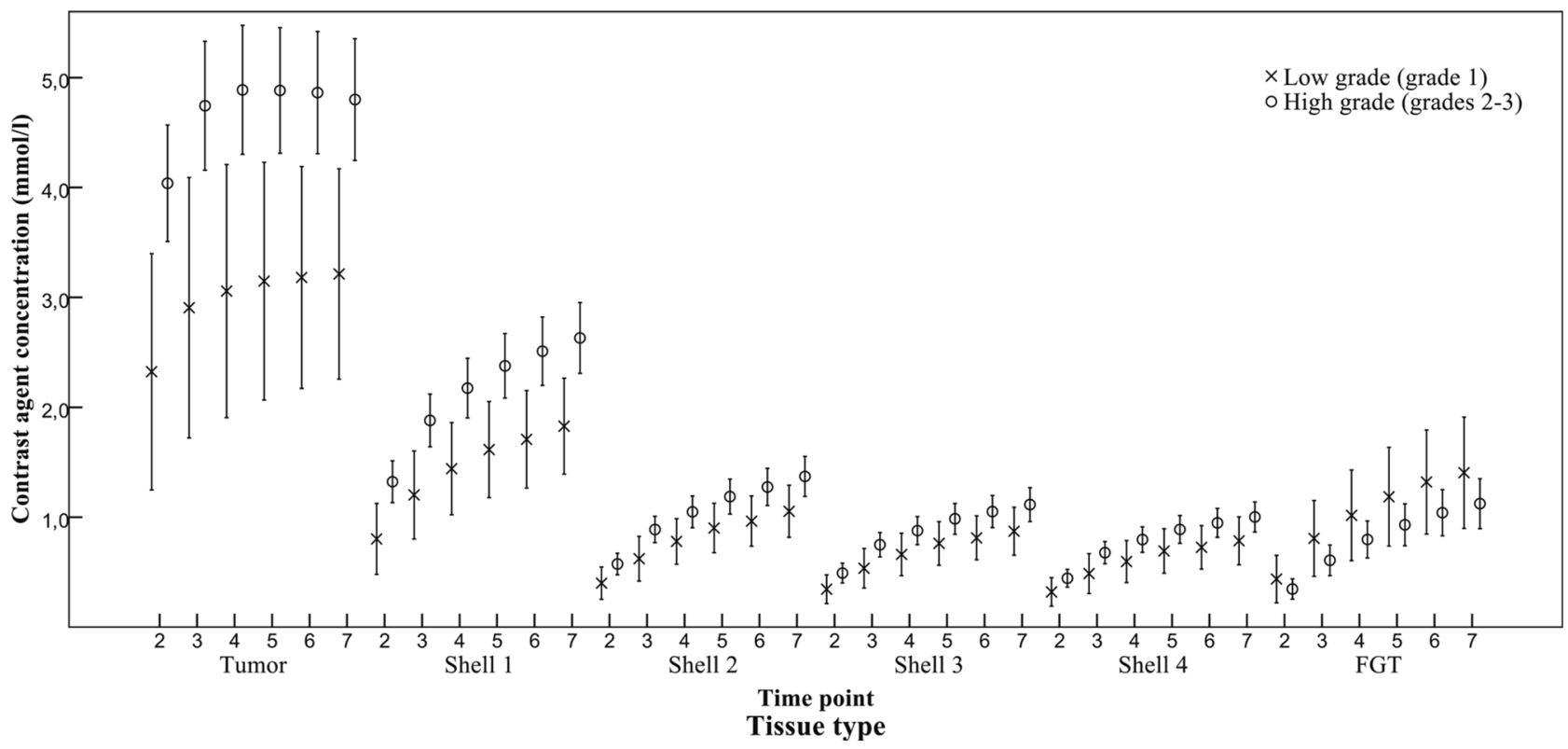

Fig. 2 Mean contrast agent concentrations (mmol/L) in breast cancers of grade 1 vs grades $2-3$. Clustered error bars at all time points. Time point 1 is in the pre-contrast phase and was not included in the measurements

correlation coefficients. Statistical significance was set at $P<0.05$ for all tests. Given the exploratory nature of this study, we did not use the Bonferroni correction for multiple comparisons.

\section{Results}

A total of 56 patients (mean age, $56.6 \pm 11.0$ years) with 56 invasive breast cancers were included in the study. The patient profiles and tumour characteristics are described in Table 1. Histopathologically, 12 patients had a low-grade (grade 1) tumour and 44 patients had a high-grade (grades 2-3) tumour.

In the intra-tumoural area, the AUC value for the concentration of contrast agent was significantly higher in the high-grade tumours (mean $27.5 \mathrm{mmol} / \mathrm{L} \times \mathrm{min}$ ) than in the low-grade tumours (mean $18.3 \mathrm{mmol} / \mathrm{L} \times \mathrm{min} ; P=0.011$ ). The mean difference was $9.14 \mathrm{mmol} / \mathrm{L} \times \min (95 \%$ confidence interval [CI] 16.1-2.20]). In the peri-tumoural area measured from the shell located closest to the tumour border (shell 1), the AUC value for the concentration of contrast agent was also significantly higher in the high-grade tumours (mean $12.3 \mathrm{mmol} / \mathrm{L} \times \mathrm{min}$ ) than in the low-grade tumours (mean $8.4 \mathrm{mmol} / \mathrm{L} \times \min ; P=0.021$ ). The mean difference was $3.82 \mathrm{mmol} / \mathrm{L} \times \min (95 \%$ CI $7.05-0.61)$.

The absolute concentrations of contrast material in both the intra-tumoural and peri-tumoural tissues were significantly higher in the high-grade tumours than in the low-grade tumours (Table 2 and Fig. 2). The high-grade tumours showed increased tissue concentrations of contrast agent at every time point examined. At the first post-contrast time point, the intra-tumoural concentration was $68.0 \%$ higher in the high-grade tumours than in the low-grade tumours. The absolute differences in the peritumoural concentrations gradually diminished in the shells situated further from the tumour border, with values of $65.0 \%, 53.8 \%, 50.0 \%$, and $48.4 \%$ in shells $1,2,3$, and 4 , respectively.

The parameters derived from the TICs correlated with the histopathological parameters (Table 3 and Fig. 3). Larger tumour size correlated with higher SER for the intra-tumoural $(r=0.348, P=0.009)$ and peri-tumoural volumes $(r=0.280, P=0.037)$. Higher tumour grade correlated with higher SER in the intra-tumoural $(r=0.470$, $P<0.001)$ and peri-tumoural volumes $(r=0.343-0.356$, $P<0.01)$. Ki67 activity correlated with the intra-tumoural $(r=0.414, P=0.002)$ and peri-tumoural $S E R$ values $(r=0.344-0.385, P<0.05)$. In addition, Ki67 activity correlated weakly with the initial percentage enhancement measured in the peri-tumoural region $(r=0.277-0.287$, $P<0.05)$. Positive oestrogen receptor status of the tumours correlated negatively with the $S E R$ values in the fibroglandular tissue of the healthy breast ( $r=-0.440, P=0.001$ ). Neither the peak percentage enhancement nor the adipose tissue measurements correlated with the clinical or histopathological markers.

Higher intra-tumoural contrast agent concentrations ( $>3.5 \mathrm{mmol} / \mathrm{L}$ at time point $2 ;>4.0 \mathrm{mmol} / \mathrm{L}$ at time point 3) achieved a positive predictive value (PPV) of over $90 \%$ 
Table 3 Associations between histopathological markers and mean signal enhancement ratio (SER) measured in different regions

\begin{tabular}{|c|c|c|c|c|c|c|c|c|c|c|c|}
\hline & $N$ & SER tumour & $P$ & SER shell 1 & $P$ & SER shell 2 & $P$ & SER shell 3 & $P$ & SER shell 4 & $P$ \\
\hline \multicolumn{12}{|l|}{ Tumour size } \\
\hline Large ( $\geq \mathrm{T} 2)$ & 22 & $0.91 \pm 0.23$ & .009 & $0.53 \pm 0.14$ & .037 & $0.45 \pm 0.18$ & .056 & $0.46 \pm 0.18$ & .071 & $0.47 \pm 0.16$ & .054 \\
\hline Small $(\leq \mathrm{T} 1 \mathrm{c})$ & 34 & $0.74 \pm 0.22$ & & $0.45 \pm 0.16$ & & $0.37 \pm 0.12$ & & $0.39 \pm 0.12$ & & $0.39 \pm 0.12$ & \\
\hline \multicolumn{12}{|c|}{ Histological grade } \\
\hline High $[2,3]$ & 44 & $0.85 \pm 0.23$ & .005 & $0.50 \pm 0.15$ & .029 & $0.42 \pm 0.15$ & .101 & $0.44 \pm 0.16$ & .108 & $0.44 \pm 0.15$ & .126 \\
\hline Low [1] & 12 & $0.64 \pm 0.21$ & & $0.40 \pm 0.13$ & & $0.34 \pm 0.10$ & & $0.36 \pm 0.11$ & & $0.36 \pm 0.11$ & \\
\hline \multicolumn{12}{|l|}{ ER } \\
\hline Positive & 48 & $0.80 \pm 0.25$ & .347 & $0.47 \pm 0.16$ & .533 & $0.39 \pm 0.15$ & .299 & $0.41 \pm 0.16$ & .214 & $0.41 \pm 0.15$ & .247 \\
\hline Negative & 8 & $0.88 \pm 0.15$ & & $0.51 \pm 0.07$ & & $0.46 \pm 0.07$ & & $0.48 \pm 0.09$ & & $0.48 \pm 0.10$ & \\
\hline \multicolumn{12}{|l|}{ PR } \\
\hline Positive & 44 & $0.79 \pm 0.23$ & .400 & $0.48 \pm 0.16$ & .965 & $0.41 \pm 0.16$ & .834 & $0.41 \pm 0.16$ & .510 & $0.41 \pm 0.15$ & .496 \\
\hline Negative & 12 & $0.86 \pm 0.29$ & & $0.48 \pm 0.13$ & & $0.40 \pm 0.11$ & & $0.44 \pm 0.12$ & & $0.45 \pm 0.13$ & \\
\hline \multicolumn{12}{|l|}{ HER2 } \\
\hline Positive & 45 & $0.87 \pm 0.25$ & .322 & $0.51 \pm 0.21$ & .539 & $0.42 \pm 0.14$ & .705 & $0.44 \pm 0.14$ & .565 & $0.44 \pm 0.15$ & .677 \\
\hline Negative & 11 & $0.79 \pm 0.24$ & & $0.47 \pm 0.14$ & & $0.40 \pm 0.15$ & & $0.41 \pm 0.15$ & & $0.42 \pm 0.14$ & \\
\hline \multicolumn{12}{|l|}{ Ki67 } \\
\hline High $(\geq 20 \%)$ & 30 & $0.90 \pm 0.22$ & .002 & $0.53 \pm 0.16$ & .003 & $0.45 \pm 0.15$ & .009 & $0.47 \pm 0.16$ & .005 & $0.47 \pm 0.15$ & .004 \\
\hline Low $(<20 \%)$ & 26 & $0.70 \pm 0.22$ & & $0.42 \pm 0.13$ & & $0.35 \pm 0.12$ & & $0.36 \pm 0.12$ & & $0.36 \pm 0.11$ & \\
\hline \multicolumn{12}{|l|}{ LNM } \\
\hline Positive & 23 & $0.88 \pm 0.23$ & .073 & $0.53 \pm 0.16$ & .049 & $0.44 \pm 0.19$ & .143 & $0.46 \pm 0.19$ & .087 & $0.46 \pm 0.18$ & .109 \\
\hline Negative & 33 & $0.76 \pm 0.23$ & & $0.45 \pm 0.14$ & & $0.38 \pm 0.10$ & & $0.39 \pm 0.10$ & & $0.40 \pm 0.11$ & \\
\hline
\end{tabular}

Fig. 3 Mean SER values in breast cancers of grade $1 \mathrm{vs}$ grades $2-3$. Clustered error bars from each tissue type measured in the first post-contrast phase

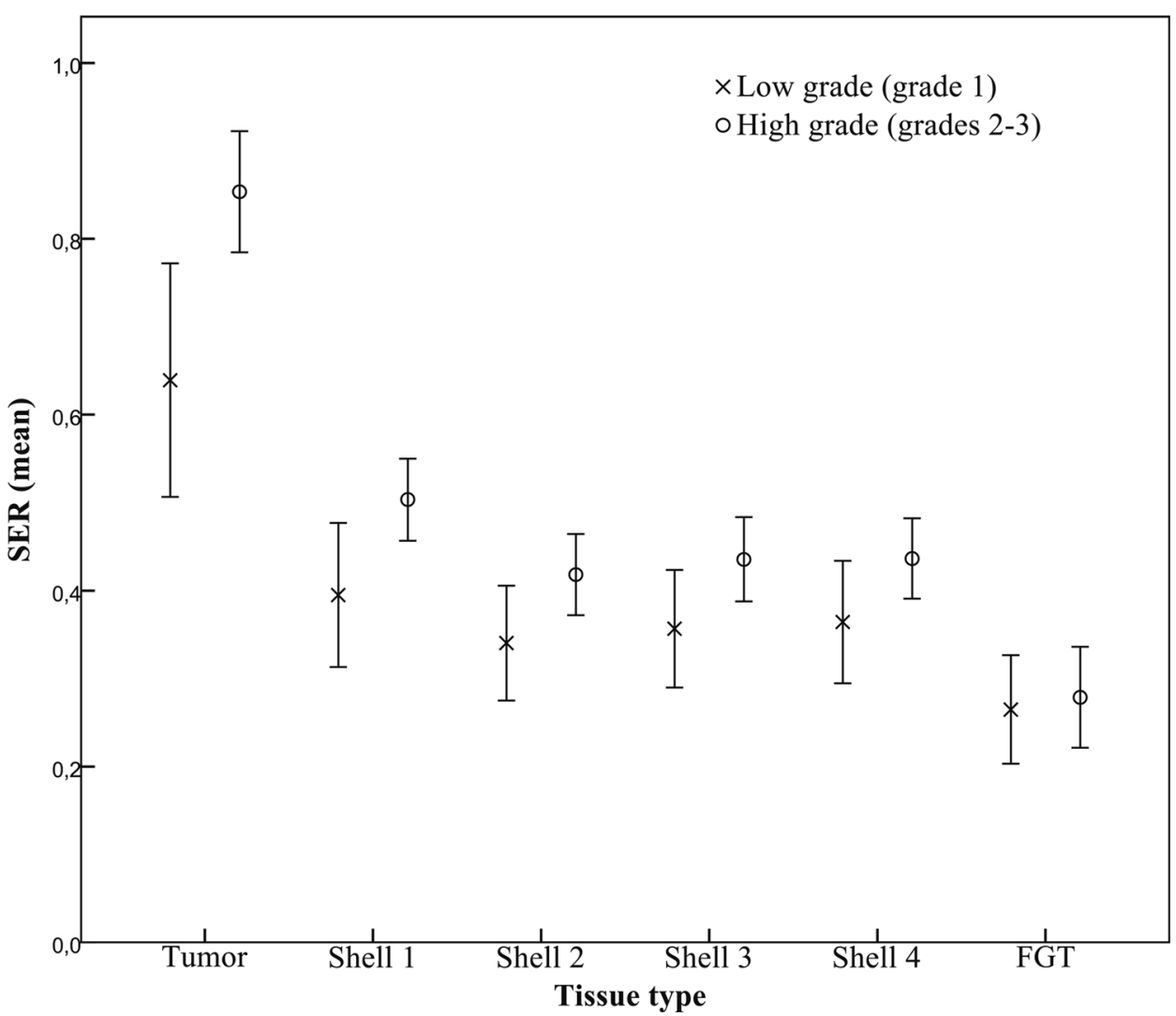


Table 4 Performance of the intra- and peri-tumoural tissue contrast agent concentration as a marker to classify tumours as low- or high-grade tumours

\begin{tabular}{lllllll}
\hline Tissue (time point) & Sensitivity (\%) & Specificity (\%) & Accuracy (\%) & PPV (\%) & NPV (\%) & $\begin{array}{l}\text { Threshold } \\
(\mathrm{mmol} / \mathrm{L})\end{array}$ \\
\hline Tumour, 2 & 65.9 & 75 & 67.9 & 90.6 & 37.5 & 3.5 \\
Tumour, 3 & 68.2 & 75 & 69.6 & 90.9 & 39.1 & 4.0 \\
Shell 1, 2 & 65.9 & 75 & 67.9 & 90.6 & 37.5 & 1.2 \\
Shell 1, 3 & 56.8 & 83.3 & 62.5 & 92.6 & 34.5 & 1.8 \\
\hline
\end{tabular}

$P P V$ positive predictive value, $N P V$ negative predictive value, Threshold the tissue contrast agent concentration used to divide tumours into low- and high-grade tumours for the detection of high-grade tumours. A similar PPV was also observed using the contrast agent concentrations in the peri-tumoural shell 1 (Table 4).

The intra-observer reproducibility of the method was high. For the intra-tumoural intensity measures, a mean Cronbach's $\alpha$ value of 0.995 was achieved between all time points. For the peri-tumoural intensity measures, mean Cronbach's $\alpha$ values of $0.974,0.995,0.998$, and 0.998 were achieved for shell 1 , shell 2 , shell 3 , and shell 4 , respectively. The Cronbach $\alpha$ values for the intra- and peri-tumoural contrast agent concentrations (0.969-0.993) were similar to those for the intensity measures. The Cronbach $\alpha$ values for the adipose and fibroglandular tissue intensities were 0.905 and 0.999 , respectively. The intraclass correlation coefficients for intra-rater consistency are shown in Table 5.

\section{Discussion}

DCE-MRI is widely used in clinical breast cancer imaging. The tumour enhancement patterns have been studied extensively and many mathematical models have been developed to represent the dynamic flow and accumulation of contrast agent. However, there are still few studies of the enhancement patterns in the peri-tumoural region. In this study, we developed

Table 5 Intra-observer reproducibility of measurements assessed with intra-class correlation coefficients. Single measures and twotailed analysis of consistency

\begin{tabular}{lcccc}
\hline & ICC & Lower & Upper & Sig \\
\hline Tumour & .986 & .949 & .996 & $<.001$ \\
Shell 1 & .968 & .886 & .991 & $<.001$ \\
Shell 2 & .990 & .965 & .997 & $<.001$ \\
Shell 3 & .994 & .978 & .998 & $<.001$ \\
Shell 4 & .996 & .984 & .999 & $<.001$ \\
Adipose & .826 & .477 & .950 & $<.001$ \\
FGT & .998 & .994 & 1.000 & $<.001$ \\
\hline
\end{tabular}

a method to measure the 3D intensity data in both the intra- and peri-tumoural regions, quantified as absolute concentrations. The kinetic parameters were also extracted from the timesignal curves. Our results show that not only did the low- and high-grade tumours differ in their intra-tumoural enhancement characteristics, but that the differences in the peri-tumoural region were also significant. Both the intra-tumoural and peritumoural contrast agent concentrations proved to have a high positive predictive value in the classification of tumours with a higher histopathological grade. These findings suggest that peritumoural pharmacokinetic parameters can be used as additional surrogate markers in future multi-parametric statistical analyses to model and predict tumour aggressiveness and prognoses, and to measure their responses to neoadjuvant treatments.

We analyzed several parameters (time intensity curves (TIC), the initial percentage enhancement $\left(E_{1}\right)$, peak percentage enhancement $\left(E_{\text {peak }}\right)$, and signal enhancement ratio $(S E R)$ ) that represent the dynamic flow and accumulation of contrast agent in breast tumours and found that high tumoural and peri-tumoural contrast agent concentrations and high SER values are associated with tumour aggressiveness. High-grade tumours showed markedly higher tissue concentrations of contrast agent than lowgrade tumours at every time point. The SER values of the intra-tumoural and peri-tumoural volumes correlated with tumour grade, size, and Ki67 activity, which are all markers for poor prognosis. Both the SER value and the absolute contrast agent concentration in the tumoural and peri-tumoural tissues may be useful as imaging indices in the characterization of tumour aggressiveness.

Currently, there is no consensus on the optimal imaging biomarkers with which to quantify the aggressiveness or prognostic characteristics of breast cancers. Earlier studies showed that even the most basic qualitative parameters (e.g. the shape of the TIC) correlate significantly with the more sophisticated quantitative perfusion parameters (e.g. $K_{\text {Trans }}, K_{\text {ep }}$, and $V_{\mathrm{e}}$ ) [28], More research on the predictive value of the existing imaging parameters, with the inclusion of new markers, is required to establish an optimal multi-parametric MRI prognostication tool. 
The peri-tumoural region has recently received increasing attention in the field of cancer imaging. The peri-tumoural area supports the wound response-like process, the inflammatory response, the increased vascular density, and the permeability of the vessels of the tumour, and consists of an extracellular matrix and various cell types [29]. Peri-tumoural markers are associated with the response to neoadjuvant chemotherapy [12, $30,31]$, hyaluronan accumulation, and lymph node metastasis $[13,14,32,33]$, pathological biomarkers [34], prognosis [35, 36], and the molecular subtype of the tumour [37]. However, the methods used to measure and extract data from the peritumoural region vary widely.

Manual segmentation has been used in many ways of even the most recent publications. Several studies have used a hand-drawn ROI placed on a single 2D slice for manual tumour delineation [12-14], whereas other studies have included multiple slices $[30,31]$ or even the whole tumour [33-35]. However, manual segmentation is time-consuming and highly operator-dependent, and thus leads to great variability between operators. Therefore, an automatic and user-independent method is required to generate more reproducible, reliable, and comparable results and to save time. Automated segmentation has been used in several studies to minimize operator error [37, 38]. The present method uses standardized 3D measurements with minimal user input, which minimizes intra- and inter-observer measurement bias, and results in excellent internal consistency. The method is also computationally undemanding, and can even be run on low-end computers.

The study limitations included the low number of patients analyzed, and the lack of access to a T1-mapping or arterial input function. However, variability is reported to be low when reference tissue models are used [22, 23, 39]. Despite these limitations, we detected significant differences between low- and high-grade tumours, highlighting the relevance of quantifying the pharmacokinetic parameters of breast tumours. Specifically, assessing the quantitative parameters in the peri-tumoural region as an adjunct to intra-tumoural assessments is a useful component of the DCE analysis.

In conclusion, characterizing breast cancer and its biomarkers as precisely as possible is important in the era of personalized medicine because treatment modalities are tailored to individuals. Our results demonstrate that the 3D segmentation model is feasible and offers an easy and standardized method of evaluating dynamic pharmacokinetic parameters, not only for tumoural areas but also for peri-tumoural areas. Low- and high-grade tumours differ significantly in their intra-tumoural and peri-tumoural enhancement characteristics. These findings should encourage the use of pharmacokinetic parameters as surrogate markers in future statistical analyses to model and predict the aggressiveness and prognosis of tumours, and to measure their responses to neoadjuvant treatments.
Funding Open access funding provided by University of Eastern Finland (UEF) including Kuopio University Hospital. This study received grants from The Cancer Society of Finland and the North Savo Regional Fund of the Finnish Cultural Foundation.

\section{Declarations}

Ethics Approval Written informed consent was obtained from all the patients before any procedure. The study was approved by the Research Ethics Board of our tertiary hospital and patients provided written informed consent. All clinical investigations were conducted according to the relevant guidelines and the principles expressed in the Declaration of Helsinki.

Conflict of Interest The authors declare no competing interests.

Open Access This article is licensed under a Creative Commons Attribution 4.0 International License, which permits use, sharing, adaptation, distribution and reproduction in any medium or format, as long as you give appropriate credit to the original author(s) and the source, provide a link to the Creative Commons licence, and indicate if changes were made. The images or other third party material in this article are included in the article's Creative Commons licence, unless indicated otherwise in a credit line to the material. If material is not included in the article's Creative Commons licence and your intended use is not permitted by statutory regulation or exceeds the permitted use, you will need to obtain permission directly from the copyright holder. To view a copy of this licence, visit http://creativecommons.org/licenses/by/4.0/.

\section{References}

1. Kuhl CK, Mielcareck P, Klaschik S. Dynamic breast MR imaging: are signal intensity time course data useful for differential diagnosis of enhancing lesions? Radiology. 1999;211:101-10.

2. Morris EA, Comstock CE, Lee CH. ACR BI-RADS® Magnetic Resonance Imaging. In: ACR BI-RADS $®$ Atlas, Breast Imaging Reporting and Data System. Reston, VA, American College of Radiology. 2013.

3. Prager GW, Poettler M. Angiogenesis in cancer. Hämostaseologie. 2012;32(2):105-14.

4. Knopp MV, Giesel FL, Marcos H, von Tengg-Kobligk H, Choyke P. Dynamic contrast-enhanced magnetic resonance imaging in oncology. Topics in Magnetic Resonance Imaging. 2001;12(4):301-8.

5. Buadu LD, Murakami J, Murayama S, Hashiguchi N, Sakai S, Masuda K, et al. Breast lesions: correlation of contrast medium enhancement patterns on MR images with histopathologic findings and tumor angiogenesis. Radiology. 1996;200(3):639-49.

6. Knopp MV, Weiss E, Sinn HP, Mattern J, Junkermann H, Radeleff $\mathbf{J}$, et al. Pathophysiologic basis of contrast enhancement in breast tumors. Journal of Magnetic Resonance Imaging. 1999; 10(3):260-6.

7. Gillies RJ, Kinahan PE, Hricak H. Radiomics: Images Are More than Pictures, They Are Data. Radiology. 2016;278(2):563-77.

8. Lambin P, Rios-Velazquez E, Leijenaar R, Carvalho S, van Stiphout, Ruud G. P. M., Granton P, et al. Radiomics: Extracting more information from medical images using advanced feature analysis. European Journal of Cancer. 2011;48(4):441-6.

9. Leithner D, Horvat JV, Marino MA, Bernard-Davila B, Jochelson MS, Ochoa-Albiztegui RE, et al. Radiomic signatures with contrast-enhanced magnetic resonance imaging for the assessment 
of breast cancer receptor status and molecular subtypes: initial results. Breast cancer research : BCR. 2019;21(1):106-11.

10. Li H, Zhu Y, Burnside ES, Drukker K, Hoadley KA, Fan C, et al. MR Imaging Radiomics Signatures for Predicting the Risk of Breast Cancer Recurrence as Given by Research Versions of MammaPrint, Oncotype DX, and PAM50 Gene Assays. Radiology. 2016;281(2):382-91.

11. Pickles MD, Manton DJ, Lowry M, Turnbull LW. Prognostic value of pre-treatment DCE-MRI parameters in predicting disease free and overall survival for breast cancer patients undergoing neoadjuvant chemotherapy. European Journal of Radiology. 2008;71(3):498-505.

12. Hattangadi J, Park C, Rembert J, Klifa C, Hwang J, Gibbs J, et al. Breast Stromal Enhancement on MRI Is Associated with Response to Neoadjuvant Chemotherapy. American Journal of Roentgenology. 2008;190(6):1630-6.

13. Kettunen T, Okuma H, Auvinen P, Sudah M, Tiainen S, Sutela A, et al. Peritumoral ADC values in breast cancer: region of interest selection, associations with hyaluronan intensity, and prognostic significance. European Radiology. 2020;30(1):38-46.

14. Choi EJ, Youk JH, Choi H, Song JS. Dynamic contrast-enhanced and diffusion-weighted MRI of invasive breast cancer for the prediction of sentinel lymph node status. Journal of Magnetic Resonance Imaging. 2020;51(2):615-26.

15. Nathaniel M Braman, Maryam Etesami, Prateek Prasanna, Christina Dubchuk, Hannah Gilmore, Pallavi Tiwari, et al. Intratumoral and peritumoral radiomics for the pretreatment prediction of pathological complete response to neoadjuvant chemotherapy based on breast DCE-MRI. Breast Cancer Research. 2017;19(1):57.

16. Sardanelli F, Boetes C, Borisch B, Decker T, Federico M, Gilbert FJ, et al. Magnetic resonance imaging of the breast: Recommendations from the EUSOMA working group. European Journal of Cancer. 2010;46(8):1296-316.

17. Schindelin J, Arganda-Carreras I, Frise E, Kaynig V, Longair M, Pietzsch T, et al. Fiji: an open-source platform for biologicalimage analysis. Nature Methods. 2012;9(7):676-82.

18. Hinkle DE, Wiersma W, Jurs SG. Applied Statistics for the Behavioral Sciences. 5th ed. Boston: Houghton Mifflin; 2003.

19. Jain A, Murty M, Flynn P. Data clustering. ACM Computing Surveys (CSUR). 1999;31(3):264-323.

20. Legland D, Arganda-Carreras I, Andrey P. MorphoLibJ: integrated library and plugins for mathematical morphology with ImageJ. Bioinformatics (Oxford, England). 2016;32(22):3532-4.

21. Ollion J, Cochennec J, Loll F, Escudé C, Boudier T. TANGO: a generic tool for high-throughput 3D image analysis for studying nuclear organization. Bioinformatics (Oxford, England). 2013;29(14):1840-1.

22. Yankeelov TE, Luci JJ, Lepage M, Li R, Debusk L, Lin PC, et al. Quantitative pharmacokinetic analysis of DCE-MRI data without an arterial input function: a reference region model. Magnetic Resonance Imaging. 2005;23(4):519-29.

23. Medved M, Karczmar G, Yang C, Dignam J, Gajewski TF, Kindler $\mathrm{H}$, et al. Semiquantitative analysis of dynamic contrast enhanced MRI in cancer patients: Variability and changes in tumor tissue over time. Journal of Magnetic Resonance Imaging. 2004;20(1):122-8.

24. Taylor AJ, Salerno M, Dharmakumar R, Jerosch-Herold M. T1 Mapping: Basic Techniques and Clinical Applications. JACC. Cardiovascular imaging. 2016;9(1):67-81.

25. Rakow-Penner R, Daniel B, Yu H, Sawyer-Glover A, Glover GH. Relaxation times of breast tissue at $1.5 \mathrm{~T}$ and $3 \mathrm{~T}$ measured using IDEAL. Journal of Magnetic Resonance Imaging. 2006;23(1):87-91.

26. Shen Y, Goerner F, Snyder C, Morelli J, Hao D, Hu D, et al. T1 Relaxivities of Gadolinium-Based Magnetic Resonance Contrast
Agents in Human Whole Blood at 1.5, 3, and 7 T. Investigative Radiology. 2015;50(5):330-8.

27. Choi EJ, Youk JH, Choi H, Song JS. Dynamic contrast-enhanced and diffusion-weighted MRI of invasive breast cancer for the prediction of sentinel lymph node status. Journal of Magnetic Resonance Imaging. 2019.

28. Yi B, Kang D, Yoon D, Jung Y, Kim K, Yim H, et al. Is there any correlation between model-based perfusion parameters and model-free parameters of time-signal intensity curve on dynamic contrast enhanced MRI in breast cancer patients? Eur Radiol. 2014;24(5):1089-96.

29. Polyak K, Kalluri R. The Role of the Microenvironment in Mammary Gland Development and Cancer. Cold Spring Harbor perspectives in biology. 2010;2(11):a003244.

30. Braman N, Prasanna P, Whitney J, Singh S, Beig N, Etesami M, et al. Association of Peritumoral Radiomics With Tumor Biology and Pathologic Response to Preoperative Targeted Therapy for HER2 (ERBB2)-Positive Breast Cancer. JAMA network open. 2019;2(4):e192561.

31. Braman NM, Etesami M, Prasanna P, Dubchuk C, Gilmore H, Tiwari $\mathrm{P}$, et al. Intratumoral and peritumoral radiomics for the pretreatment prediction of pathological complete response to neoadjuvant chemotherapy based on breast DCE-MRI. Breast cancer research : BCR. 2017;19(1):57.

32. Zhang S, Yi S, Zhang D, Gong M, Cai Y, Zou L. Intratumoral and peritumoral lymphatic vessel density both correlate with lymph node metastasis in breast cancer. Scientific reports. 2017;7(1):40364.

33. Liu C, Ding J, Spuhler K, Gao Y, Serrano Sosa M, Moriarty M, et al. Preoperative prediction of sentinel lymph node metastasis in breast cancer by radiomic signatures from dynamic contrastenhanced MRI. Journal of Magnetic Resonance Imaging. 2019;49(1):131-40.

34. Shin HJ, Park JY, Shin KC, Kim HH, Cha JH, Chae EY, et al. Characterization of tumor and adjacent peritumoral stroma in patients with breast cancer using high-resolution diffusionweighted imaging: Correlation with pathologic biomarkers. European Journal of Radiology. 2016;85(5):1004-11.

35. Wu J, Li B, Sun X, Cao G, Rubin DL, Napel S, et al. Heterogeneous Enhancement Patterns of Tumor-adjacent Parenchyma at MR Imaging Are Associated with Dysregulated Signaling Pathways and Poor Survival in Breast Cancer. Radiology. 2017;285(2):401-13.

36. Cheon H, Kim HJ, Kim TH, Ryeom H, Lee J, Kim GC, et al. Invasive Breast Cancer: Prognostic Value of Peritumoral Edema Identified at Preoperative MR Imaging. Radiology. 2018;287(1):68-75.

37. Chen J, Zhang Y, Chan S, Chang R, Su M. Quantitative analysis of peri-tumor fat in different molecular subtypes of breast cancer. Magnetic Resonance Imaging. 2018;53:34-9.

38. Zhou J, Zhang Y, Chang K, Lee KE, Wang O, Li J, et al. Diagnosis of Benign and Malignant Breast Lesions on DCE-MRI by Using Radiomics and Deep Learning With Consideration of Peritumor Tissue. Journal of magnetic resonance imaging : JMRI. 2019.

39. Yang C, Karczmar GS, Medved M, Aytekin O, Zamora M, Stadler WM. Reproducibility Assessment of a Multiple Reference Tissue Method for Quantitative DCE-MRI Analysis. Magnetic resonance in medicine: official journal of the Society of Magnetic Resonance in Medicine/Society of Magnetic Resonance in Medicine. 2009;61(4):851-9.

Publisher's Note Springer Nature remains neutral with regard to jurisdictional claims in published maps and institutional affiliations. 